\title{
Geographic patterns of seed trait variation in an invasive species: how much can close populations differ?
}

\author{
Erola Fenollosa ${ }^{1,2}$ [ $\cdot$ Laia Jené$^{1} \cdot$ Sergi Munné-Bosch ${ }^{1,2}$
}

Received: 1 August 2020 / Accepted: 11 June 2021 / Published online: 3 July 2021

(c) The Author(s) 2021, corrected publication 2021

\begin{abstract}
Seeds play a major role in plant species persistence and expansion, and therefore they are essential when modeling species dynamics. However, homogeneity in seed traits is generally assumed, underestimating intraspecific trait variability across the geographic space, which might bias species success models. The aim of this study was to evaluate the existence and consequences of interpopulation variability in seed traits of the invasive species Carpobrotus edulis at different geographical scales. We measured seed production, morphology, vigour and longevity of nine populations of C. edulis along the Catalan coast (NE Spain) from three differentiated zones with a human presence gradient. Geographic distances between populations were contrasted against individual and multivariate trait distances to explore trait variation along the territory, evaluating the role of bioclimatic variables and human density of the different zones. The analysis revealed high interpopulation variability that was not explained by geographic distance, as regardless of the little distance between some populations $(<0.5 \mathrm{~km})$, significant differences were found in several seed traits. Seed production, germination, and persistence traits showed the strongest spatial variability up to $6000 \%$ of percent trait variability between populations, leading to differentiated C. edulis soil seed bank dynamics at small distances, which may demand differentiated strategies for a cost-effective species management. Seed trait variability was influenced by human density but also bioclimatic conditions, suggesting a potential impact of increased anthropogenic pressure and climate shifts. Geographic interpopulation trait variation should be included in ecological models and will be important for assessing species responses to environmental heterogeneity and change.
\end{abstract}

Keywords Bioclimatic variables $\cdot$ Carpobrotus edulis $\cdot$ Geographic distance $\cdot$ Longevity $\cdot$ Mediterranean

\section{Introduction}

Seeds, the reproductive units of flowering plants, constitute one of the main factors defining the persistence and expansion of species and therefore play a crucial role in determining species success (Saatkamp et al. 2014; Gioria and Pyšek 2016). Moreover, seed persistence and dispersal have deep consequences for a species genetic diversity and adaptative

Communicated by Casey P. terHorst.

Erola Fenollosa

efenollosa@ub.edu

1 Department of Evolutionary Biology, Ecology and Environmental Sciences, Faculty of Biology, Universitat de Barcelona, Avinguda Diagonal 643, 08028 Barcelona, Spain

2 Institute of Research in Biodiversity (IRBio-UB), Universitat de Barcelona, Avinguda Diagonal 643, 08028 Barcelona, Spain dynamics (Tigano and Friesen 2016). A wide range of morphological and physiological traits enable seeds to coordinate persistence and germination timing, as well as dispersion maximization through different agents (Poschlod et al. 2013; Long et al. 2015; Saatkamp et al. 2019). Variations in seed morphological traits may determine differential dispersion strategies that can favour adaptation and colonization of different habitats (Lamberti-Raverot et al. 2019). For instance, high seed production, small seed mass, and rapid germination have been associated with higher invasive success in novel habitats (Pyšek and Richardson 2007; Gioria and Pyšek 2017; Gioria et al. 2018). In species with orthodox seeds, viable seeds may enter dormancy, a temporal suppression of germination under favourable conditions, thanks to their ability to tolerate considerable desiccation (Bewley et al. 2013). Higher dormancy rates may facilitate the formation of a persistent seed bank, which may substantially contribute to determining the invasion potential, given the role of seed banks as sources of propagules, genetic diversity, 
and in spreading the risk of germination failure over time (Gioria et al. 2019). Complex biochemical regulation prevents seeds to germinate, resisting for several years at the soil seed bank (Kucera et al. 2005). Seed longevity may differ between species, ranging from just a few months to more than 2000 years in Phoenix dactylifera (Sallon et al. 2008). Even within a species, seed longevity may vary based on several factors such as moisture content, relative humidity, oxygen pressure, and temperature (Walters et al. 2005). Longevous seeds may increase the capacity of a species to persist in a particular site and increase the impact of invasive species due to increased needs of post-eradication management (Gioria et al. 2016). Altogether, seed morphology, production, viability, germination, biochemistry, and persistence may have important implications in species success (Saatkamp et al. 2019), as they offer an approximated reflect of the soil seed bank dynamics (Gioria and Pyšek 2016).

Despite all well-known seed processes and the role of seeds on species persistence and expansion, the degree of variability that a species may show in seed production, viability, germination and persistence between different closer and distant populations may be underestimated. Intraspecific variation of seed traits still constitutes a major concern in the research agenda for seed-trait functional ecology (Saatkamp et al. 2019) as the reliability of trait-based mechanistic models depends on the representation of intraspecific trait variation, that may drive to enormous differences (Albert et al. 2011). The understanding of the causes, consequences, and degree of intraspecific variability may impact ecological models of species response to global change and species potential distribution (Saatkamp et al. 2019; Snell et al. 2019).This may be particularly important for understanding and modelling the biological invasion process, where modelling seed persistence and expansion across the geographic space (i.e., across different populations) is crucial for risk assessment and management. In this way, Jarić et al. (2019) highlighted that spatial variation in seed traits may be one of the contributors to the uncertainty when trying to model invasive species impact and develop management strategies. Not only for improving ecological modelling, but variability on an invasive species impact may require the development of differential management strategies (Januchowski-Hartley et al. 2018).

To what extend a species seed traits dissimilarity increases with geographic distance is crucial in assumptions taken for species persistence and expansion modelling. However, the geographic variation of seed traits for a species, i.e., seed traits interpopulation variability, may come from different sources. Environmental heterogeneity, which includes climatic and habitat variability, is considered the main driver of intraspecific variability (Kuppler et al. 2020) and may strongly contribute to interpopulation variability with the more similar populations under the more similar environmental conditions. It may be therefore assumed that geographic distance together with environmental distance (environmental dissimilarity) and habitat heterogeneity may determine interpopulation variability. Bioclimatic dissimilarity was key in determining seed thickness variation of the invasive plant Taraxacum officinale in five differentiated Chilean populations with a rainfall gradient (MolinaMontenegro et al. 2018), but for example, the study from Bogdziewicz et al. (2019) found that Quercus petraea seed production may vary across populations independently of the geographic (and bioclimatic) distance between them. The bioclimatic conditions that promote increased interpopulation variability are not clear and may not be universal. It has been proposed that abiotic conditions near the limit of tolerance of a species can exert a role towards both increased or reduced intraspecific variability (Caruso et al. 2017; Helsen et al. 2017). Not only the climatic conditions a species may encounter in a location but also habitat heterogeneity may contribute to variability in the environment and modulate species intraspecific variability (De Smedt et al. 2018). Urbanization is a land-use change form characterized by increased human impacts as a consequence of their increased presence, fragmentation of natural habitats, intensive land transformation and an increase in impervious surface land cover thus reducing the spatial extent for vegetation (Irwin and Bockstael 2007; Williams et al. 2015). Urban environments create a wide range of novel habitats such as industrial areas or waste dumps that may constrain species performance and community assemblance (Irwin and Bockstael 2007), and therefore increased intraspecific variability in urban species traits may be expected. This may be particularly important in invasive species, that benefit from those novel habitats within urban areas and are subjected to novel environmental pressures that may induce high intraspecific variation, especially regarding plant seed traits that compromise species persistence and expansion (Gaertner et al. 2017). Moreover, multiple introductions and therefore the increased propagule pressure in urbanized areas with higher human presence may also propitiate higher intraspecific variability in invasive species (Smith et al. 2020).

The invasive species Carpobrotus edulis (L.) N. E. Br. (Aizoaceae) is a mat-forming trailing succulent perennial native from South Africa that has been introduced in all continents, strongly impacting Mediterranean regions (Vilà et al. 2008). C. edulis was introduced in Europe as a valuable ornamental plant but also for soil and dune stabilization (Preston and Sell 1988). C. edulis impacts reside on its ecosystem engineer capacity to modify the surrounding environment by altering physicochemical soil properties (Molinari et al. 2007; Novoa et al. 2013) and decrease native plant richness and functional diversity (Jucker et al. 2013; Novoa and González 2014). C. edulis has small hard-coated reniform orthodox seeds, which can generate a permanent 
soil seed bank that may contribute to the species impact and persistence in the invaded communities (Chenot et al. 2014; Fenollosa et al. 2020). Carpobrotus sp. seeds may persist in the soil seed bank for more than 5 years allowing quick reinvasion many years after removal (up to 8 years) (Affre 2011; Ruffino et al. 2015). In Catalonia, C. edulis constitutes a major biodiversity conservation concern because it inhabits and impacts most of the coastal zones. However, the shoreline has been massively urbanized, leaving few spaces for vegetation. Across the Catalan coast, some zones have been urbanized more than others. In the northern part, the zone of Cap de Creus (CA) is the eastern foothill of the Pyrenees. It includes marine and terrestrial protected areas and constitutes an area of high biological, geological, and landscape quality with large extensions of woody sclerophyll Mediterranean vegetation. Forty kilometres south, the zone of middle Costa Brava (CB, the Baix Empordà county) constitutes an area where tourism is more intense and combines large zones of established plant communities coexisting with urbanizations, campings and touristic complexes. Finally, getting closer to Barcelona, the Maresme (M) coast is intensely urbanized with large agricultural areas leaving almost no virgin plant communities. Among those three zones, there is a strong gradient in human presence in terms of population density per squared kilometre. High phenotypic plasticity has been observed in this invader regarding biomass allocation, morphological variations and photoprotective responses (Traveset et al. 2008; Roiloa et al. 2014, 2016; Fenollosa et al. 2017). However, seed traits variability across different geographic scales in the differentiated three zones that $C$. edulis inhabits in Catalonia has not been studied and may determine differential invasive vigour in this highly plastic invasive species.

The aims of this study were to (a) test the existence of interpopulation variability in plant traits associated with sexual reproduction of the invasive species $C$. edulis at different geographical scales, contrasting closer $(<4 \mathrm{~km})$ and distant ( $>40 \mathrm{~km}$ ) populations, and (b) determine the role of geographic distance, bioclimatic conditions and human presence on interpopulation variability. We hypothesized that (1) the variability found within a population (intrapopulation) may be lower than the variability between different populations (interpopulation) in distant populations $(>40 \mathrm{~km})$, but not in closer populations $(<4 \mathrm{~km})$, so geographic distance may have a role determining similarity between populations due to similar environmental conditions. Moreover, we hypothesized that (2) distant populations may differ in key seed traits that may determine differentiated population dynamics such as: seed production, viability, germination and dormancy, as a consequence of local adaptation. This would hamper invasive species dynamics modelling, as a model obtained from the mean values of different populations would not reflect the actual species dynamics. Finally, we expected (3) increased seed traits variability in zones with increased human presence due to the increased propagule pressure and the variability in microhabitat conditions, and higher seed traits variability in sites where individuals of this species might suffer more stressful conditions according to previous studies (lower temperatures on winter and lower water availability on summer) (Fenollosa et al. 2017). To test these hypothesis, we evaluated the interpopulation variability of nine populations of $C$. edulis and measured different parameters describing different aspects of the invasive species reproductive traits such as: seed morphology, production, viability, germination, biochemistry and persistence analysed the variability within and between the different populations at contrasted geographic distances in relation with their degree of human presence and bioclimatic variables.

\section{Materials and methods}

\section{Plant populations and seed collection}

Nine populations of $C$. edulis along the Catalan coast (NE Spain) were selected considering different geographic distances (Fig. 1a). Within three distant zones (> $40 \mathrm{~km}$ ) (Cap de Creus, Costa Brava and Maresme) where C. edulis is abundant, three populations were sampled based on closeness $(<4 \mathrm{~km})$ and enough fruit production to measure all seed traits. In average, the three $C$. edulis populations in the CA zone have a population density of 39 habitants $\mathrm{km}^{-2}$, whereas CB has 188 habitants $\mathrm{km}^{-2}$ and M 285 habitants $\mathrm{km}^{-2}$ (Geostat Eurostat 2011). These differences in human presence and habitat availability (higher in the CA zone) may also influence the surrounding vegetation, besides terrain characteristics. Unfortunately, there is no data regarding introduction time or invasion front within the territory.

From each population of a minimal area of $500 \mathrm{~m}^{2}$, within the period of natural seed rain, 600 fruits were collected to ensure representativeness. Fruits were opened and all the obtained seeds were pooled (more than 10,000 seeds per population). Seeds were kept at darkness and room temperature until analysis (3-4 weeks). Multiple ecological significant traits related to seed production, seed morphology, viability, germination, persistence, and biochemistry were measured and are summarized in Online Resource 2.

\section{Bioclimatic data}

Nineteen bioclimatic variables were obtained from the WorldClim 2 database at 0.5 arcmin resolution $\left(\sim 1 \mathrm{~km}^{2}\right)$ (Fick and Hijmans 2017). The bioclimatic variables represent annual trends (e.g., mean annual temperature, annual 
Fig. 1 a Relative location of the nine studied populations (filled circles) of C. edulis distributed in three differentiated zones:

Maresme (M), Costa Brava

(CB) and Cap de Creus (CA).

b Results of multidimensional scaling analysis (MDS) evaluating differences in nine seed traits among studied populations. Traits indicated in grey have significant $(P<0.01)$ contribution population variability. Ellipses represent $95 \%$ of confidence intervals. $P$-values correspond to PERMANOVA results for Zone and Population (nested in Zone) factors (a)
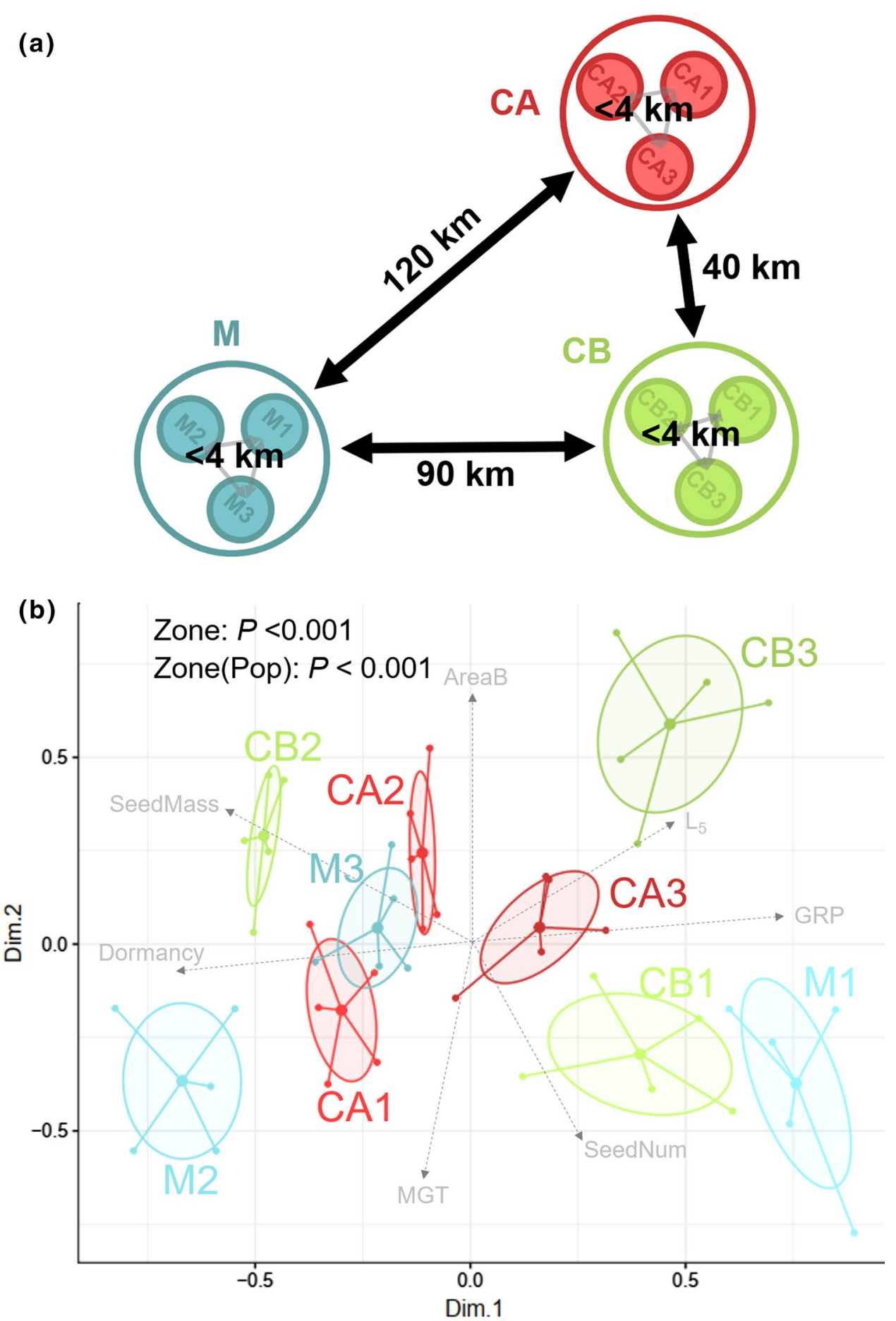

precipitation) seasonality (e.g., annual range in temperature and precipitation) and extreme or limiting environmental factors (e.g., temperature of the coldest and warmest month, and precipitation of the wet and dry quarters). Climatic information of all studied populations can be found in the Online Resource 1.

\section{Seed morphology and production}

Five fruits from each population were used to determine seed production in terms of fruit weight (FruitWeight), total seed weight (SeedsWeight) and total seed number (SeedNum). From each fruit, five seeds were randomly selected to measure morphometric seed parameters such as 
seed area, perimeter, major diagonal, minor diagonal, and thickness, considering basal and lateral scaled captures of $4 \times$ magnification under a binocular loupe leading to the following parameters from basal capture: basal seed area (AreaB), basal seed perimeter (PerimB), major basal diagonal (MajorB), minor basal diagonal (MinorB), and from lateral capture: lateral seed area (AreaL), lateral seed perimeter (PerimL), major lateral diagonal (MajorL), minor lateral diagonal (MinorL). All measurements were performed with ImageJ software (Wayne Rasband, Java, National Institutes of Health, Bethesda, USA). The five seeds per fruit were averaged and considered individual replicates, with one value per fruit for each seed morphology trait, to contrast these values with seed production traits per fruit unit.

Five replicates of fifty seeds were used to estimate seed mass (SeedMass) and the dry seed mass (DrySeedMass) after four days at $70{ }^{\circ} \mathrm{C}$. Seed mass and dry seed mass were obtained by dividing the obtained fresh and dry weight respectively by seed number.

\section{Seed vigour: germination, viability and longevity}

Seed germination for all populations was performed as described in Fenollosa et al. (2020) with $12 \mathrm{~h}$ photoperiod and temperature alternance between $25{ }^{\circ} \mathrm{C}$ during day and $10{ }^{\circ} \mathrm{C}$ at night. Germination was assessed every three days on petri plaques with fifty seeds each with five replications per population. Total germination percentage (GRP) was estimated after curve saturation at 70 days. Different seed germination indexes regarding speed and synchrony were estimated: mean germination time (MGT) (days), mean germination rate (MGR) (seeds day $\left.{ }^{-1}\right)$, germination speed percentage (GSP) (\%), uncertainty index (UNC) (bits), synchronization index (SYN) (from 0 to 1 ), germination variance (VGT), germination standard deviation (SDG), coefficient of variation (CVG) $(\%)$, and $\mathrm{T}_{50}$ (The time required for $50 \%$ germination). The germination uncertainty index (UNC) is based on the Shannon indexes and estimates the uncertainty in predicting the informal entropy associated with the distribution of the germination relative frequency in bits units (information units) (Labouriau and Valadares 1983). The synchronization index (SYN) assesses the synchrony of one seed with other included in the same replication $(\mathrm{SYN}=0$ when at least two seeds germinate one each time and $\mathrm{SYN}=1$ when the germination of all the seeds occurs at the same time) (Ranal and Santana 2006). Five replications per population, including fifty seeds per replication were used for the germination test.

Seed viability was assessed using the viability tetrazolium (Triphenyl tetrazolium chloride) test following the method and embryo viability classification described in Fenollosa et al. (2020) for $C$. edulis. In brief, after $24 \mathrm{~h}$ of seed imbibition, seeds were delicately pierced to ensure tetrazolium penetration. Seeds with $0.1 \%$ tetrazolium (Sigma-Aldrich, Steinheim, Germany) were incubated for $48 \mathrm{~h}$ at $40{ }^{\circ} \mathrm{C}$ before viability assessment. Six different categories were used to classify embryo status: viable (totally stained), weakly (pale stained), patchy (partially stained), patchyweakly (partially pale stained), dead (white embryo), and aborted (no embryo found). The sum of weakly, patchy and patchy-weakly seeds constitutes low vigour seeds without survivance probabilities (Dying). The percentage of dormant seeds was estimated as the difference between viable but not germinated seeds.

Seed longevity was assessed through an accelerated ageing test as described by Fenollosa et al. (2020) for C. edulis on which seeds were subjected to $55{ }^{\circ} \mathrm{C}$ under high relative humidity conditions (80-90\%) for different timings that lead to a progressive viability loss. Viability was totally loss for all populations after $196 \mathrm{~h}$ under the accelerated ageing conditions and $\mathrm{L}_{5}$ (resistance to deterioration), $\mathrm{L}_{50}$ (medium longevity) and $\mathrm{L}_{95}$ (lethal ageing time) (Number of hours to lose 5,50 , and $95 \%$ viability respectively) were used as longevity estimators. Five replicates per population, including 250 seeds per replication were used for viability and longevity assessment.

\section{Seed biochemistry: antioxidants and seed water content}

During ageing, seeds are subjected to oxidative stress that may be counterbalanced with antioxidants compromising seed longevity (Bailly 2004). Among antioxidant systems, tocochromanols are lipophilic antioxidants particularly abundant in seeds that were found to be essential in determining seed longevity (Sattler et al. 2004). Tocochromanols are lipid-soluble antioxidant molecules and its accumulation in seeds has been described to be critical in maintaining seed viability by protecting lipids from oxidation during germination and early seedling growth (Sattler et al. 2004). Five replicates of $100 \mathrm{mg}$ of seed samples from each population were ground in liquid nitrogen using a mix ball and extracted with cold methanol containing $0.01 \%$ butylated hydroxyltoluene using ultrasonication. After centrifuging at $14,000 \mathrm{~g}$ for $10 \mathrm{~min}$ at $4{ }^{\circ} \mathrm{C}$, the supernatant was collected and the pellet re-extracted with the same solvent until it was colourless; then, supernatants were pooled and filtered with $0.22 \mu \mathrm{m}$ and transferred to high-performance liquid chromatography (HPLC) vials. Tocochromanols were separated isocratically in a normal-phase HPLC system and quantified with a fluorescent detector, as described by Amaral et al. (2005). Quantification was based on the results obtained from the fluorescence signal and compared to that of a calibration curve made with authentic standards (Sigma-Aldrich, Steinheim, Germany). Gamma-tocopherol ( $\gamma$-Toc), alpha-tocopherol $(\alpha-$ Toc) and their sum (Toc) were detected in $C$. edulis seeds. 
Seed water content (WC), and imbibed seed water content (WC_Imb) were measured with five replicates of fifty seeds per population as (fresh - dry weight $\times 100) \times$ dry weight $^{-1}$ from seeds and imbibed seeds for $24 \mathrm{~h}$ respectively. Dry weight was obtained after four days at $70{ }^{\circ} \mathrm{C}$.

\section{Data analysis}

All analyses were performed in R 4.0.3 (R Core Team, 2020) using the following $R$ packages: vegan (Oksanen et al. 2019), mctoolsr (https://github.com/leffj/mctoolsr/), multcomp (Hothorn et al. 2008), agricolae (Mendiburu and Yaseen 2020) and VCA (Schuetzenmeister and Dufey 2020). Germination parameters were calculated using GerminaR package (Lozano-Isla et al. 2019). The ggplot2 R package (Wickham 2016) and SigmaPlot 10.0 (Systat, USA) were used for plotting.

\section{Aim A: interpopulation vs. intrapopulation variability}

To evaluate the degree of interpopulation variability (aim A), the percent potential variability of each trait between populations, hereafter 'percent trait variability between populations' was calculated as the mean percent difference when contrasting all measured values from one population to another

To test the similarity of the different populations and contrast the hypothesis that intrapopulation variability is lower than interpopulation variability (hypothesis 1 ), nine traits were selected. Trait selection was based on their ecological relevance and significant contribution to explaining the global dataset variance in the multivariate space using multidimensional scaling. The nine selected traits represent the 6 trait types considered: basal seed area and seed mass (seed morphology), seed number per fruit (seed production), seed viability (viability), seed germination and mean germination time (germination), $\gamma$-tocopherol (biochemistry), resistance to deterioration $\mathrm{L}_{5}$ and dormancy (persistence). Permutational multivariate analysis of variance (PERMANOVA) was used to contrast zones and populations (nested in zone) using a multidimensional approach with 999 permutations with scaled data, using the adonis() function from the vegan $\mathrm{R}$ package, using the scaled trait values as a response variable and Zone and Zone(Pop) as predictor variables. The function calc_pairwise_permanovas() from the $\mathrm{R}$ package mctools $r$ was used to evaluate differences between zones and populations. As measured traits resulted in independent replicates, PERMANOVA results were tested after multiple dataset randomizations to confirm the obtained statistic results.

To evaluate the populations' differentiation in individual seed traits and test the hypothesis that distant populations may differ in key seed traits that may determine differentiated population dynamics (hypothesis 2), a nested ANOVA was used to evaluate the significance of the factors Zone and Population (nested in Zone) in all measured seed traits using the trait values as a response variable and Zone and Zone(Pop) as predictor variables. ANOVA assumptions were tested with Shapiro-Wilk and Levene tests for normality and homoscedasticity. The Tukey test was used as a post-hoc method using the multcomp and agricolae $R$ packages to evaluate differences between populations for all traits.

\section{Aim B: the role of geographic distance, bioclimatic conditions and human density on interpopulation variability}

To determine the main source of variation and determine the role of geographic distance, bioclimatic conditions and human density on interpopulation variability (aim B), relative variance decomposition at the population and zone-levels was performed for the nine selected traits using the anovaMM() function from the VCA R package. For each trait (response variable), variance was decomposed and quantified across sampling scales and expressed as a percentage of the total variance explained by Zone, Zone(Pop) and residual variance (predictor variables).

To determine the role of geographic distance, Mantel tests were run to evaluate the correlation between distance matrices of individual seed traits and geographic distance using the mantel() function from the vegan $\mathrm{R}$ package, using 999 permutations. This allowed testing the second part of hypothesis 1 regarding the role of geographic distance determining populations' similarity.

To assess the role of bioclimatic variables and human density in interpopulation variability within a zone (hypothesis 3 ), the betadisper() function from the vegan $\mathrm{R}$ package was used to obtain the 'distance to centroid', hereafter: zonal variability, for the multidimensional data of each zone, this is, the distance of each data point to the centroid obtained using the scaled data of the 9 selected traits of each zone. This parameter was used to compare trait variation within each of the three distant zones. An ANOVA was performed to test significant differences between the response variable zonal variability in the different Zones. The Tukey test was used as a post-hoc method. Zonal variability was correlated with the data of all 19 bioclimatic variables in each population and the data of human density in each Zone, using the cor() function in R. Due to the high number of comparisons and to avoid type I error, $P$-values were adjusted using the FDR method (Benjamini and Hochberg 1995). 


\section{Results}

\section{Strong interpopulation variability in seed production, germination and persistence}

The three geographically distant zones across the Catalan coast (Cap de Creus, Costa Brava and Maresme) have an annual precipitation from 558 to $621 \mathrm{~mm}$ and an annual mean temperature around the $16{ }^{\circ} \mathrm{C}$ (Online Resource 1). Despite the similar environmental conditions, the multivariate analysis of the populations revealed significant effects for zones and populations $(P<0.001)$ (Fig. 1b). The relative position of the different populations on the multidimensional scaled plot, reveal that some distant populations such as M3 and CA1 are more similar than close populations such as M3 and M1 in terms of the 9 seed traits considered (Fig. 1b).

Significant differences between zones and populations were found considering almost all measured seed parameters when contrasting not only distant but also near populations (Fig. 2b, Online Resource 2). Considering the three geographically distant zones, seeds from the populations at Costa Brava (CB) showed significantly higher seed mass and viability percentages compared to Maresme and Cap de Creus but high variability was found between populations within each zone (Fig. 2b, d). Significantly lower germination percentages were found in Cap de Creus seeds although big variability was found between populations, from 25 to $80 \%$ of germinated seeds (Fig. 2E). Costa Brava seeds showed significantly lower mean germination time (about 2 weeks), whereas the mean germination time for seeds from other populations raised to 30 days (Fig. 2f). The highest percentage of dormancy was found in a Costa Brava population but high variability found within populations, from 20 to $60 \%$ of dormant seeds (Fig. 2i).

Considering the different near and distant populations, post-hoc tests of seed traits revealed that even close populations have different seed area, seed mass, total seeds per fruit, seed viability, germination percentage, mean
Fig. 2 Trait values (mean $\pm S E$, $n=5$ ) for the nine relevant seed traits in all nine C. edulis populations from three distant zones: Cap de Creus (CC), Costa Brava (CB) and Maresme (M). Different capital letters reflect differences between zones, whereas different lowercase letters reflect significant differences between populations

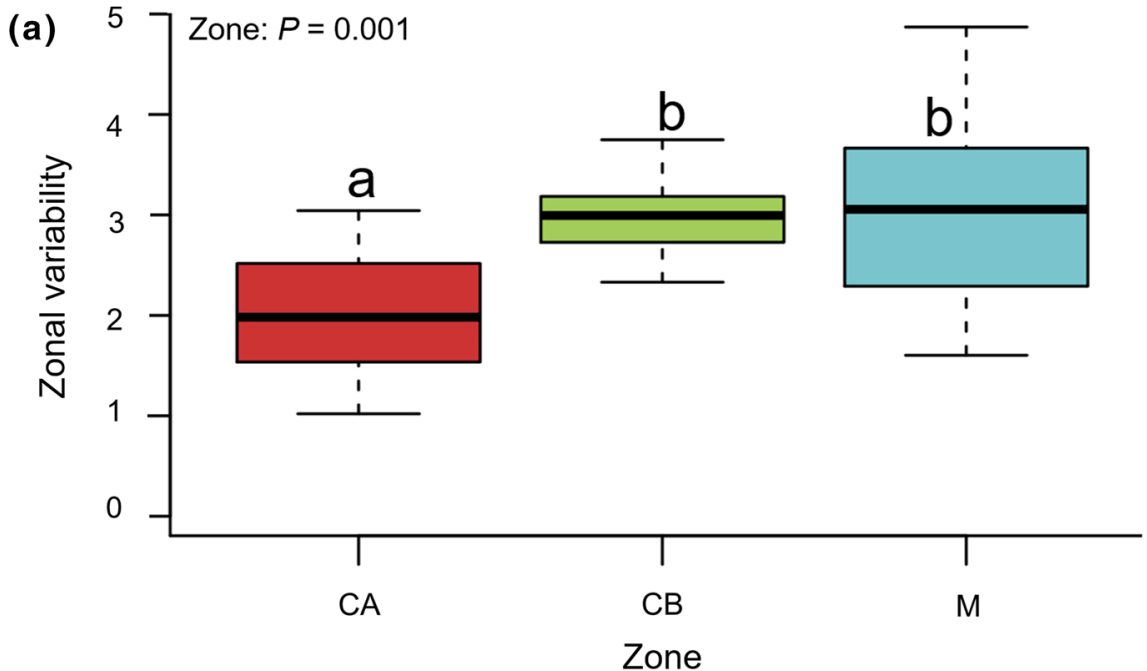

(b) Correlations with zonal variability

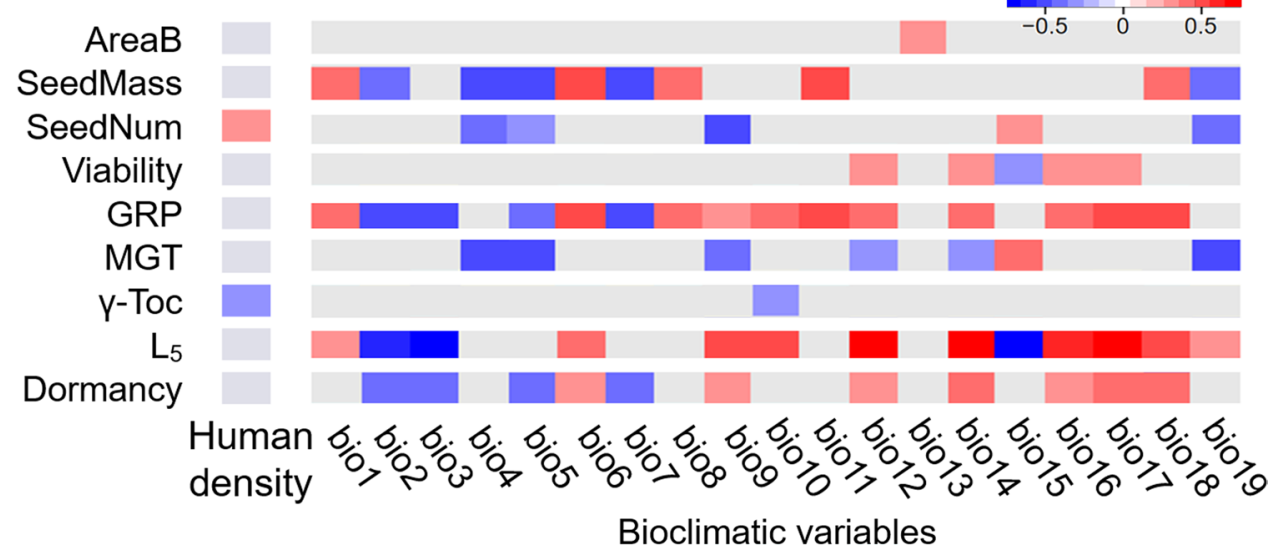


germination time, $\boldsymbol{\gamma}$-tocopherol, $\mathrm{L}_{5}$ and dormancy percentages (Fig. 2). The smallest seeds were found in M1 with an average seed mass of $19.44 \pm 0.82 \mu \mathrm{g}$ and a basal seed area of $0.81 \pm 0.04 \mathrm{~mm}^{2}$ whereas the heaviest seeds were found in a population located at $c a .40 \mathrm{~km}, \mathrm{CB} 2$, with $28.04 \pm 0.01 \mu \mathrm{g}$, and a basal area of $1.00 \pm 0.02 \mathrm{~mm}^{2}$ (Fig. 2a, b). Besides seed morphology, near $C$. edulis populations also differed in seed production, viability, germination, biochemistry and persistence (Fig. 2, Online Resource 2). Total seeds per fruit maximum difference between populations were found between two close populations: CB3 and CB1 and ranged from $57.73 \pm 42.80$ to $2478.45 \pm 345.32$ seeds respectively. Despite the huge standard error, those differences resulted significant (Fig. 2c), showing a more than 40 times higher average seed production at less than $1 \mathrm{~km}$ of distance. Seed viability ranged from 71 to $88 \%$ but germination ranged from 22 to $80 \%$ across populations, arising differences in the percentage of dormant seeds between populations (Fig. 2i). The most abundant tocochromanol found, $\boldsymbol{\gamma}$-tocopherol, also differed significantly between near populations (Fig. 2g). Seeds $\gamma$-tocopherol concentration ranged from $5.43 \pm 0.44$ in $\mathrm{CC} 2$ to $7.55 \pm 0.30 \mathrm{mg} \mathrm{gDW}^{-1}$ in CB1 (Fig. $2 \mathrm{~g}$ ), whereas no significant differences were found in its successor, $\alpha$-tocopherol, that remained around $0.27 \pm 0.01 \mathrm{mg} \mathrm{gDW}^{-1}$ (Online Resource 2). Longevity analysis revealed differences between zones with M2 showing strongly decreased seed resistance to deterioration in terms of $\mathrm{L}_{5}$ (Fig. 2h).

The variance structure revealed differences in the proportion of variance explained by the different factors across traits (Fig. 3a). Differences among populations captured on average, $35-90 \%$ of the total variability, while the zone factor added an additional 0-20\% to the total proportion of variability explained. However, only two traits—seed viability and mean germination time-showed a proportion of variability explained by the zone factor superior to $15 \%$ (Fig. 3a).

When traits variability was translated into percent trait variability between populations, different patterns considering the different traits type were observed (Fig. 3b). The strongest percent trait variability between populations was found in total seed number per trait (up to $6000 \%$ mean values of change) whereas the smallest percent trait variability was found in seed morphology traits (up to $20 \%$ mean values). Seed germination and persistence showed also a disparate variation from 0 to $400 \%$. Finally, $\gamma$-tocopherol content and seed viability showed a small percent variation (under 20\%) (Fig. 3b).

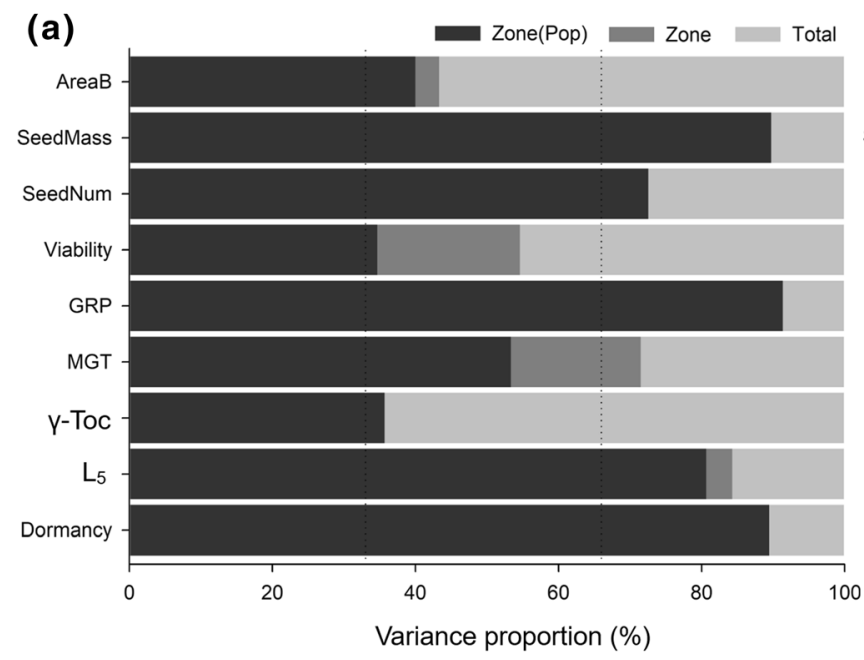

(c)

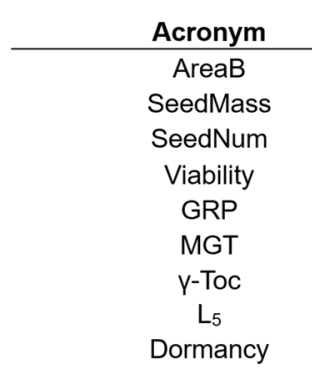

Trait Basal seed area $\left(\mathrm{mm}^{2}\right)$ Seed mass ( $\mathrm{mg}$ ) Total seed number per fruit Percentage of viable embryos (\%) Total germination percentage (\%) Mean germination time (days) Gamma tocopherol (mg/gDW)

Resistance to deterioration (hours) Dormancy percentage (\%)

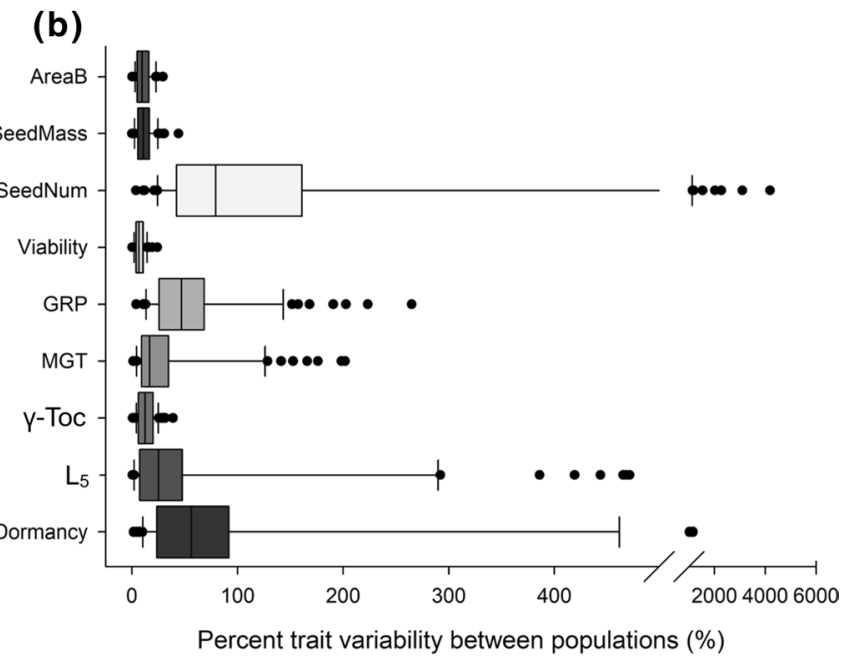

Percent trait variability between populations (\%)

Mantel Test with geographic distance

$$
\begin{gathered}
P=0.048, \mathrm{R}_{M}=0.077 \\
\mathrm{NS} \\
\mathrm{NS} \\
P=0.029, \mathrm{R}_{\mathrm{M}}=0.099 \\
P=0.042, \mathrm{R}_{\mathrm{M}}=0.087 \\
P=0.033, \mathrm{R}_{\mathrm{M}}=0.094 \\
\mathrm{NS} \\
P=0.001, \mathrm{R}_{\mathrm{M}}=0.247 \\
\mathrm{NS}
\end{gathered}
$$

Fig. 3 a Relative variance decomposition at the population and zonelevels for the different traits. The 33 and $66 \%$ thresholds are given by the dashed lines for the nine selected seed traits of $C$. edulis. b Boxplot of percent trait variation for the nine selected seed traits of
C. edulis between diferent populations. c Acronyms, complete trait names, and Mantel Test results for the correlation between geographic variation with trait variation. $N S$ non-significant, $R_{M}$ mantel statistic 


\section{Geographic distance, bioclimatic variables and human density influence trait variability}

Correlation of geographic with trait's distances across populations did not result in any strong correlation in most of the studied traits, as revealed by the Mantel tests (Fig. 3c). Poor or non-significant correlation coefficients for the Mantel test were also obtained when contrasting geographic distance with the multivariate scaled seed traits distance between populations (Online Resource 2).

Despite the high geographic variability found in almost all seed traits, this was not homogeneous among zones. The variability found between the near populations in the area of Cap de Creus, the zone with lower human density, was significantly lower than the variation between near populations in the other zones (Fig. 4a). When decomposing zonal variability among traits, seed number variability within a zone showed a positive significant correlation with human density, suggesting that populations in areas with a higher human presence may have stronger variability in this trait (Fig. 4b). The accumulation of $\gamma$-tocopherol correlated inversely to human density, suggesting that the lower human density, the higher $\gamma$-tocopherol variability. Seed traits variability was contrasted with the 19 bioclimatic variables from the WorldClim model and revealed strong and significant correlations with most traits (Fig. 4b). The bioclimatic variables with the strongest significative correlations $(P<0.05$, $R^{2}>0.6$ and $\left.R^{2}<-0.6\right)$ with traits variability were: bio3 (isothermality), bio12 (annual precipitation), bio14 (precipitation of the driest month), bio15 (precipitation seasonality), bio16 (precipitation of the wettest quarter) and bio17 (precipitation of the driest quarter). Among those variables, some showed a clear negative correlation with most traits' variability. Mean diurnal (bio2) and annual range (bio7),
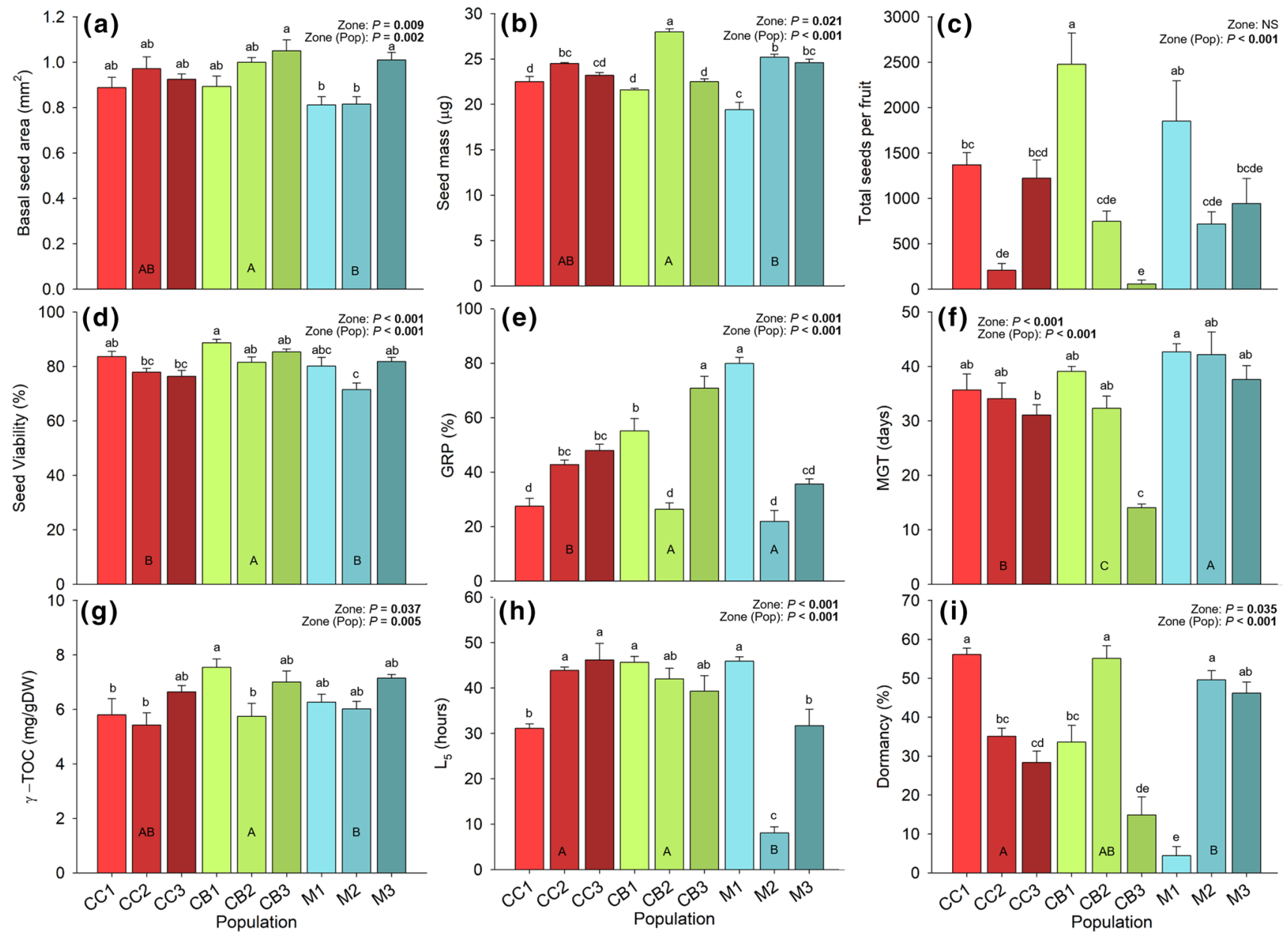

Fig. 4 a Comparative of the zonal variability estimated as the distances to zonal centroid for the different populations at the zones: Cap de Creus (CA), Costa Brava (CB) and Maresme (M) using the nine selected seed traits. Different letters reflect significant differences between zones. b Human density and bioclimatic variables correla- tions with zonal variability (centroid distance) of the nine seed traits of $C$. edulis among the different populations. Coloured correlations are significant and blue to red palette represents the $R^{2}$ coefficient following the colour key. Complete bioclimatic variables names can be found in http://www.worldclim.org/bioclim 
isothermality (bio3), temperature seasonality (bio4) and the maximum temperature of the warmest month (bio5) correlated inversely with zonal traits variability. In the other hand, annual mean temperature (bio1), minimum temperature of the coldest month (bio6), the temperature of the wettest (bio8) and the coldest (bio11) year quarters and precipitation of the wettest (bio16), driest (bio17) and warmest year (bio18) quarters showed strong positive correlations with zonal traits variability (Fig. 4b). Higher variability was therefore observed in zones with higher temperature and precipitation among the year, and lower temperature variation within days and seasons. The strongest correlation found was a positive correlation between the precipitation of the driest month (bio14) and resistance to deterioration $\left(\mathrm{L}_{5}\right)$ variability $\left(P<0.05, R^{2}=-0.71\right)$. This trait showed also strong significant $\left(P<0.05, R^{2}>0.63\right)$ correlations with different bioclimatic variables: isothermality (bio3) and precipitation seasonality (bio15) correlated with decreased $\mathrm{L}_{5}$ variability, whereas annual precipitation (bio12), precipitation of the wettest (bio16) and the driest (bio17) year quarters correlated with increased $\mathrm{L}_{5}$ variability (Fig. $4 \mathrm{~b}$ ). Altogether, the higher $L_{5}$ variability was found in sites with higher and less variable precipitation among the year.

\section{Discussion}

\section{Variability in seed traits in geographically near populations}

Intraspecific variation is considered as a key factor mediating the effects of individuals on community structure and ecosystem functioning (Des Roches et al. 2018). However, intraspecific variation may be found not only between individuals but also between different populations or even regions (Evangelista et al. 2019). In this study we obtained percentages of variation across the geographic space, revealing the existence of surprisingly high seed traits interpopulation variability in an invasive species, regardless of geographic distance.

Seed traits types showed a differential degree of trait variation, suggesting different traits sensitivity, supporting hypothesis 2 that expected high interpopulation variability in key seed traits determining soil seed bank dynamics such as seed production, germination, and persistence. Among the trait groups that showed the strongest geographic variationseed production, seed germination and seed persistenceseed production is known to vary strongly in some species in the temporal scale (i. e., interannual variability) (Moreira et al. 2014; Bogdziewicz et al. 2019). Few studies have considered spatial variation in seed production traits, but for example, the study from Wright et al. (2005) evaluated both temporal and spatial variation and pointed out that spatial variation in seed fall was greater than temporal variation among years for all the 108 studied species from Panama for 15 years. This spatial variation in seed production could be a result of the environmental conditions, genetic diversity or also adaptation to pollinators and seed predator regimes (Linhart et al. 2014; Moreira et al. 2014; Mitchell et al. 2017). Besides high seed production geographic variability, germination, and persistence traits showed also a high spatial variability. Mother plant water stress has been described to compromise seed viability and dormancy in Astragalus nitidiflorus growing in the Mediterranean area (Segura et al. 2015). As fruit ripening occurs during the harsh Mediterranean summers in C. edulis, mother plant water stress could be the cause of the observed variability in seed traits among populations. However, only mean germination time showed an inverse significant correlation with annual precipitation and precipitation of the driest month, suggesting that summer low water availability may impact variability of mean germination time for this species. Intrapopulation variability in germination is common as a result of genetic factors and climate variability during seed ripening (Baskin and Baskin 1973). Strong regional variability in seed germination was found in Nothofagus glauca when contrasting 9 populations from two differentiated regions in Chile revealing higher variability within regions than between them (Santelices Moya et al. 2017). Germination variability together with high intraspecific variability in persistence traits may maximize plant long-term fitness through a bed-hedging strategy, ensuring regeneration at different time scales (Tielbörger et al. 2011; Huang et al. 2016).

Geographic variability in the different analysed traits related to $C$. edulis sexual reproduction may have implications on the dynamics of the invasive populations. C. edulis populations located at less than $1 \mathrm{~km}$, showed differential seed production, seed mass, seed germination percentage, mean germination time, seed longevity, and dormancy percentages, and these differences between populations may suppose differential soil seed bank dynamics at the different sites. The population of Carrer del Golf (CB2) for example, showed a similar seed trait's profile to Far de s'Arenella (CC1) and Can Teixidor (M2), located at 40 and almost $100 \mathrm{~km}$ of distance respectively. Those three populations have high proportions of dormant viable seeds (Figs. 2, 3). Besides, the $c a .1000$ seeds produced per fruit in those populations contribute to forecast the formation of a soil seed bank at the described locations. Contrarily, the population Puig Sa Guilla (CB3) that showed a small seed production (less than 100 seeds per fruit), have the highest germination percentages and the lowest germination time, revealing low dormancy ratios, similarly to the population at Ca l'Antic (M1). Variability in achene traits was assessed in Fallopia $x$ bohemica in different French populations, revealing differential dispersion strategies between populations, which 
may facilitate the colonization of contrasting environments (Lamberti-Raverot et al. 2019). Similarly, the seed traits interpopulation variation in $C$. edulis determines different strategies regarding seed bank dynamics. Some of the analysed $C$. edulis populations showed a persistence strategy with the formation of a soil seed bank whereas others showed a more expansive strategy (high germination and low dormancy rates). Interpopulation trait variation, including traits related to sexual reproduction, should be included in ecological models to predict species distribution (Albert et al. 2011; Banitz 2019; Snell et al. 2019) but also to predict invasive species potential distribution and impact.

\section{Contributors to seed traits variability: geographic distance, bioclimatic conditions and human density}

When unravelling the determinants of intraspecific seed trait variability in C. edulis, population, bioclimatic conditions and human density seem to play a role. The C. edulis populations separation determines differences in traits related to the sexual reproduction independently of the geographic distance between them, as revealed the absence of strong correlations at the Mantel test, the high percentage of variance explained by the population factor and the differences in mean trait values between close populations, revealing that there are no identical populations in terms of seed traits as the nearest populations can be the less similar. We, therefore, reject the hypothesis 1 that expected low differences between close populations, in contrast with higher interpopulation variability between distant populations. This strong interpopulation variability regardless of geographic distance may hinder species modelling and may have different causes.

As hypothesized (hypothesis 3), human density may be one of the causes of increased interpopulation seed production variability in $C$. edulis. A recent study with the clonal invader Plantago lanceolata revealed that in the invasive range, repeated, long-distance, human-mediated introductions promoted high genetic diversity (Smith et al. 2020). As in P. lanceolata, the human presence in the invasive range may also play a role in $C$. edulis variability, as the variability in $C$. edulis seed traits found between the different close populations was different between the three contrasted zones with a human density gradient. The area with the lowest human presence, the zone of Cap de Creus, was the zone where the smallest variation in $C$. edulis seed traits was registered. The increased number and variability of novel habitats generated by urbanization (Irwin and Bockstael 2007) and disturbance (Kumordzi et al. 2019), and also the increased chance of repeated human-mediated introductions (Smith et al. 2020) may contribute to the increased seed traits variability found in $C$. edulis zones with intense anthropogenic pressure. In this way, the meta-analysis conducted by Williams et al. (2015) already exposed that the observed variability in urban plant traits, including seed traits, is linked to the consistency and strength of urban stressors. This result, in concordance with the framework presented by Gaertner et al. (2017), suggests that anthropogenic pressure in urban areas may exacerbate the invasive species impacts not only by acting as launching sites but also by increasing its intraspecific variability of species within urban environments. In the case of $C$. edulis, the study of Lechuga-Lago et al. (2017) revealed that there is a strong link between invasion and urban areas, as urbanisation assist $C$. edulis invasion, which in turn increase the impact of urbanisation. This study suggests that $C$. edulis invasion of urban soils is facilitated due to soil degradation, which allows the establishment of this species and hinders some native species establishment. Besides, higher human density may promote increased propagule pressure in species with horticultural interests, which increases the probability of a successful establishment (Willi and Van 2019). The particular bioclimatic conditions that the species encounter in each population also contributes to seed traits spatial variability, as most of the analysed seed traits correlated with bioclimatic variables. Environmental variables influenced for instance Fagus sylvatica seed production variability, as the mean temperature was found to be correlated with seed production (Bogdziewicz et al. 2019). Moreover, the high seed traits variability among invasive Chilean Taraxacum officinale populations appeared to be highly correlated with variation in rainfall (Molina-Montenegro et al. 2018) revealing the importance of environmental conditions and seasonality in determining seed traits variability. Recently, the study of global gradients for intraspecific trait variation with almost 3000 species revealed pronounced associations with climate (Kuppler et al. 2020). Despite the idiosyncrasy of species-specific associations across gradients, in general, lower variation was observed in colder areas and higher in drier areas. In concordance with this study, seed traits variation in $C$. edulis was generally higher in zones with higher temperatures. However, increased interpopulation variability was observed with higher precipitation along the year. This contradicts the hypothesis 3 that intrapopulation variability may be higher with lower temperatures in winter and low water availability in summer, which constitute two known stressful periods for species inhabiting the Mediterranean climates, including $C$. edulis (Fenollosa et al. 2017). Stressful conditions may not induce seed trait variation in C. edulis. In this way, our study supports the stress-reduced variability hypothesis that states that variability decreases with abiotic conditions that may generate stress (Klopfer and MacArthur 1961). Changes in the global bioclimatic conditions may have an impact on seed traits variability of $C$. edulis. However, reduced pluviometry expected in a global change framework (Christensen et al. 2007) may exhort contrary effects on seed traits variability in contrast to increased 
temperatures. Further research must be addressed to assess global change effects on $C$. edulis seed traits variability.

\section{Geographic intraspecific variation in invasive species: management implications}

Knowledge regarding the intrapopulation variation across the geographic space may enhance our ability to predict ecological impacts of invasive species across different invaded areas. The found variability in seed traits between $C$. edulis populations may have important implications for this invasive species management. The work developed by Loddo et al. (2019) that evaluated variability in the invasive species Abutilon theophrasti seed traits demonstrated that achieving a better knowledge of interpopulation variability can allow specific control strategies to be designed, facilitating the development of new management tools. Likewise, germination rates of the Alexandra palm were found to potentially better inform of management strategies for the control of this species (Wen 2019). Considering the obtained results in $C$. edulis, the differential seed behaviour may determine differential requirements in soil management after eradication. Populations such as $\mathrm{CB} 2, \mathrm{CC} 1$ and M2, with high seed dormancy rates may require management cost in the long term after eradication as recruitment may be expected from the soil seed bank. The reason why those populations, with similar bioclimatic conditions and human density to the others within their zone, behave this way is uncertain and may require further research. However, the ability to form persistent seed banks might contribute substantially to determine the invasion potential of alien plants in their new distribution ranges, given the role of seed banks as sources of propagules, genetic diversity, and in spreading the risk of germination failure over time (Gioria et al. 2019). Therefore, the inclusion of soil seed bank dynamics analysis during prioritization of eradication zones may help to develop costeffective management strategies, by increasing investment in post-eradication management in populations with high seed longevity and prioritizing populations with strong seed production, as those populations may have an increased invasive potential (Pyšek and Richardson 2007).

Our study quantified the interpopulation variability in traits related to the sexual reproduction $C$. edulis between near $(<4 \mathrm{~km})$ and distant populations $(>40 \mathrm{~km})$ and revealed high geographic variability in seed production, germination and persistence traits. The observed variability in the $C$. edulis seed production, germination and dormancy rates in some populations suggested differential soil seed bank dynamics that may require differential strategies for cost-effective management. Seed traits interpopulation variability was found to be influenced by bioclimatic conditions suggesting a potential impact of climate shifts. Moreover, increased human density correlated with higher interpopulation variability in seed production suggesting a role of high anthropogenic pressure in seed traits variability which may contribute to exacerbate the invasive species impacts in urban areas. Interpopulation trait variation should be included in ecological models and will be important for assessing invasive species responses to environmental heterogeneity and global change.

Supplementary Information The online version contains supplementary material available at https://doi.org/10.1007/s00442-021-04971-2.

Acknowledgements We are very grateful to Parc Científic and Servei de Camps Experimentals of the Universitat de Barcelona for their technical assistance. We are indebted to Vadim Pisarenco and Ignasi Arqués for their collaboration in germination tests and their help in collecting seeds. We thank Marina Pérez-Llorca for her valuable suggestions on data analysis. The authors declare that they have no conflict of interest.

Author contribution statement EF and SMB conceived the idea and $\mathrm{EF}, \mathrm{LJ}$ and SMB designed the experimental design. EF and LJ performed the experiments and analysed the samples. EF performed statistical analyses and prepared the manuscript. LJ and SMB revised the manuscript. All authors read and approved the final manuscript.

Funding Open Access funding provided thanks to the CRUE-CSIC agreement with Springer Nature. This work was supported by Generalitat of Catalunya with the ICREA Academia award and the 2017 SGR 980 project given to SMB. EF is a recipient of an FPU fellowship from the Spanish Ministry of Education (FPU 15/06666).

Open Access This article is licensed under a Creative Commons Attribution 4.0 International License, which permits use, sharing, adaptation, distribution and reproduction in any medium or format, as long as you give appropriate credit to the original author(s) and the source, provide a link to the Creative Commons licence, and indicate if changes were made. The images or other third party material in this article are included in the article's Creative Commons licence, unless indicated otherwise in a credit line to the material. If material is not included in the article's Creative Commons licence and your intended use is not permitted by statutory regulation or exceeds the permitted use, you will need to obtain permission directly from the copyright holder. To view a copy of this licence, visit http://creativecommons.org/licenses/by/4.0/.

\section{References}

Affre L (2011) Ecological and evolutionary insights from plant invasions: the case of Carpobrotus spp. (Aizoaceae) in the Mediterranean Basin. In: Berhardt LV (ed) Advances in medicine and biology. Nova Science Publishers, New York

Albert CH, Grassein F, Schurr FM, Vieilledent G, Violle C (2011) When and how should intraspecific variability be considered in trait-based plant ecology? Perspect Plant Ecol, Evol Syst 13:217225. https://doi.org/10.1016/j.ppees.2011.04.003

Amaral JS, Casal S, Torres D, Seabra RM, Oliveira BPP (2005) Simultaneous determination of tocopherols and tocotrienols in hazelnuts by a normal phase liquid chromatographic method. Anal Sci 21:1545-1548. https://doi.org/10.2116/analsci.21.1545

Bailly C (2004) Active oxygen species and antioxidants in seed biology. Seed Sci Res 14:93-107. https://doi.org/10.1079/SSR2004159 
Banitz T (2019) Spatially structured intraspecific trait variation can foster biodiversity in disturbed, heterogeneous environments. Oikos 128:1478-1491. https://doi.org/10.1111/oik.05787

Baskin JM, Baskin CC (1973) Plant population differences in dormancy and germination characteristics of seeds: heredity or environment? Am Midl Nat 90:493-498. https://doi.org/10.2307/ 2424478

Benjamini Y, Hochberg Y (1995) Controlling the false discovery rate: a practical and powerful approach to multiple testing. J Royal Stat Soc Series B (methodological) 57(1):289-300. https://doi.org/10. 1111/j.2517-6161.1995.tb02031.x

Bewley JD, Bradford KJ, Hilhorst HWM, Nonogaki H (2013) Seeds: physiology of development, germination and dormancy, 3rd edn. Springer, New York. https://doi.org/10.1007/978-1-4614-4693-4

Bogdziewicz M, Szymkowiak J, Fernández-Martínez M, Peñuelas J, Espelta J (2019) The effects of local climate on the correlation between weather and seed production differ in two species with contrasting masting habit. Agric for Meteorol 268:109-115. https://doi.org/10.1016/j.agrformet.2019.01.016

Caruso CM, Martin RA, Sletvold N, Morrissey MB, Wade MJ, Augustine KE, Carlson SM, MacColl ADC, Siepielski AM, Kingsolver JG (2017) What are the environmental determinants of phenotypic selection? A meta-analysis of experimental studies. Am Nat 190:363-376. https://doi.org/10.1086/692760

Chenot J, Affre L, Passetti A, Buisson E (2014) Consequences of iceplant (Carpobrotus) invasion on the vegetation and seed bank structure on a Mediterranean island: response elements for their local eradication. Acta Botanica Gallica 161(3):301-308. https:// doi.org/10.1080/12538078.2014.910473

Christensen JH, Hewitson B, Busuioc A, Chen A, Gao X, Held R et al (2007) Regional climate projections. In: Solomon S, Qin D, Manning M, Chen Z, Marquis M, Averyt KB, Tignor M, Miller HL (eds) Climate Change 2007: the physical science basis. Contribution of Working Group I to the Fourth Assessment Report of the Intergovernmental Panel on Climate Change. Cambridge University Press, Cambridge

De Smedt P, Ottaviani G, Wardell-Johnson G, Sykora K, Mucina L (2018) Habitat heterogeneity promotes intraspecific trait variability of shrub species in Australian granite inselbergs. Folia Geobot 53:133-145. https://doi.org/10.1007/s12224-018-9311-x

Des Roches S, Post DM, Turley NE, Bailey JK, Hendry AP, Kinnison MT, Schweitzer JA, Palkovacs EP (2018) The ecological importance of intraspecifc variation. Nat Ecol Evol 2:57-64. https://doi. org/10.1038/s41559-017-0402-5

Evangelista C, Olden JD, Lecerf A, Cucherousset J (2019) Scaledependent patterns of intraspecific trait variations in two globally invasive species. Oecologia 189:1083-1094. https://doi.org/10. 1007/s00442-019-04374-4

Fenollosa E, Munné-Bosch S, Pintó-Marijuan M (2017) Contrasting phenotypic plasticity in the photoprotective strategies of the invasive species Carpobrotus edulis and the coexisting native species Crithmum maritimum. Physiol Plant 160:185-200. https://doi.org/ 10.1111/ppl.12542

Fenollosa E, Jené L, Munné-Bosch S (2020) A rapid and sensitive method to assess seed longevity through accelerated aging in an invasive plant species. Plant Methods 16(64):1-11. https://doi.org/ 10.1186/s13007-020-00607-3

Fick SE, Hijmans RJ (2017) WorldClim 2: new 1-km spatial resolution climate surfaces for global land areas. Int J Climatol 37:43024315. https://doi.org/10.1002/joc.5086

Gaertner M, Wilson JRU, Cadotte MW, MacIvor JS, Zenni RD, Richardson DM (2017) Non-native species in urban environments: patterns, processes, impacts and challenges. Biol Invasions 19:34613469. https://doi.org/10.1007/s10530-017-1598-7
Gioria M, Pyšek P (2016) The legacy of plant invasions: changes in the soil seed bank of invaded plant communities. Bioscience 66(1):40-53. https://doi.org/10.1093/biosci/biv165

Gioria M, Pyšek P (2017) Early bird catches the worm: germination as a critical step in plant invasion. Biol Invasions 19:1055. https:// doi.org/10.1007/s 10530-016-1349-1

Gioria M, Pyšek P, Osborne BA (2018) Timing is everything: does early and late germination favor invasions by herbaceous alien plants? J Plant Ecol 11(1):4-16. https://doi.org/10.1093/jpe/ rtw105

Gioria M, Le Roux JJ, Hirsch H, Moravcová L, Pyšek P (2019) Characteristics of the soil seed bank of invasive and non-invasive plants in their native and alien distribution range. Biol Invasions 21:2313-2332. https://doi.org/10.1007/s10530-019-01978-y

Helsen K, Acharya KP, Brunet J, Cousins SAO, Decocq G, Hermy M, Kolb A, Lemke IH, Lenoir J, Plue J, Verheyen K, De Frenne P, Graae BJ (2017) Biotic and abiotic drivers of intraspecific trait variation within plant populations of three herbaceous plant species along a latitudinal gradient. BMC Ecol 17:38. https://doi.org/ 10.1186/s12898-017-0151-y

Hothorn T, Bretz F, Westfall P (2008) Simultaneous inference in general parametric models. Biom J 50(3):346-363. https://doi.org/ 10.1002/bimj.200810425

Huang Z, Liu S, Bradford KJ, Huxman TE, Venable DL (2016) The contribution of germination functional traits to population dynamics of a desert plant community. Ecology 97:250-261. https://doi. org/10.1890/15-0744.1

Irwin EG, Bockstael NE (2007) The evolution of urban sprawl: evidence of spatial heterogeneity and increasing land fragmentation. Proc Natl Acad Sci USA 104:20672-20677. https://doi.org/10. 1073/pnas.0705527105

Januchowski-Hartley SR, Adams VM, Hermoso V (2018) The need for spatially explicit quantification of benefits in invasive-species management. Conserv Biol 32:287-293. https://doi.org/10.1111/ cobi.13031

Jarić I, Heger T, Castro Monzon F, Jeschke JM, Kowarik I, McConkey KR, Pyšek P, Sagouis A, Essl F (2019) Crypticity in biological invasions. Trends Ecol Evol 34(4):291-302. https://doi.org/10. 1016/j.tree.2018.12.008

Jucker T, Carboni M, Acosta AT (2013) Going beyond taxonomic diversity: deconstructing biodiversity patterns reveals the true cost of iceplant invasion. Divers Dist 19:1566-1577. https://doi. org/10.1111/ddi.12124

Klopfer PH, MacArthur RH (1961) On the causes of tropical spe-cies diversity: niche overlap. Am Nat 95:223-226. https://doi.org/10. 1111/j.1654-1103.2003.tb02138.x

Kucera B, Cohn M, Leubner-Metzger G (2005) Plant hormone interactions during seed dormancy release and germination. Seed Sci Res 15(4):281-307. https://doi.org/10.1079/SSR2005218

Kumordzi BB, Aubin I, Cardou F et al (2019) Geographic scale and disturbance influence intraspecific trait variability in leaves and roots of North American understorey plants. Funct Ecol 33:17711784. https://doi.org/10.1111/1365-2435.13402

Kuppler J, Albert CH, Ames GM, Armbruster WS, Boenisch G, Boucher FC, Campbell DR, Carneiro LT, Chacón-Madrigal E, Enquist BJ, Fonseca CR, Gómez JM, Guisan A, Higuchi P, Karger DN, Kattge J, Kleyer M, Kraft NJB, Larue-Kontić AAC, Lázaro A, Lechleitner M, Loughnan D, Minden V, Niinemets Ü, Overbeck GE, Parachnowitsch AL, Perfectti F, Pillar VD, Schellenberger Costa D, Sletvold N, Stang M, Alves-dos-Santos I, Streit H, Wright J, Zych M, Junker RR (2020) Global gradients in intraspecific variation in vegetative and floral traits are partially associated with climate and species richness. Glob Ecol Biogeogr. https://doi. org/10.1111/geb.13077

Labouriau LG, Valadares MEB (1983) The germination of seeds. OEA, Washington 
Lamberti-Raverot B, Piola F, Vallier F, Gardette V, Puijalon S (2019) Achene traits involved in the water dispersal of the invasive Fallopia $\times$ bohemica complex: variability and dispersal strategies. Flora 251:88-94. https://doi.org/10.1016/j.flora.2019.01.002

Lechuga-Lago Y, Novoa A, Le Roux JJ, Gonzalez L (2017) Understanding the influence of urbanization on invasibility: Carpobrotus edulis as an exemplar. Biol Invasions 19:3601-3611. https:// doi.org/10.1007/s10530-017-1593-Z

Linhart YB, Moreira X, Snyder MA, Mooney KA (2014) Variability in seed cone production and functional response of seed predators to seed cone availability: support for the predator satiation hypothesis. J Ecol 102:576-583. https://doi.org/10.1111/1365-2745.12231

Loddo D, Bozic D, Calha IM, Dorado J, Izquierdo J, Šćepanović M, Barić K, Carlesi S, Leskovsek R, Peterson D, Vasileiadis VP, Veres A, Vrbničanin S, Masin R (2019) Variability in seedling emergence for European and North American populations of $\mathrm{Abu}$ tilon theophrasti. Weed Res 59:15-27. https://doi.org/10.1111/ wre. 12343

Long RL, Gorecki MJ, Renton M, Scott JK, Colville L, Goggin DE, Commander LE, Westcott DA, Cherry H, Finch-Savage WE (2015) The ecophysiology of seed persistence: a mechanistic view of the journey to germination or demise. Biol Rev Camb Philos Soc 90:31-59. https://doi.org/10.1111/brv.12095

Lozano-Isla F, Benites-Alfaro O, Pompelli MF (2019) GerminaR: an R package for germination analysis with the interactive web application “GerminaQuant for R.” Ecol Res 34(2):339-346. https://doi. org/10.1111/1440-1703.1275

Mendiburu F, Yaseen M (2020) Agricolae: statistical procedures for agricultural research. R package version 1.4 .0 . https://myase en208.github.io/agricolae/https://cran.r-project.org/package $=$ agricolae

Mitchell J, Johnston IG, Bassel GW (2017) Variability in seeds: biological, ecological, and agricultural implications. J Exp Bot 68(4):809-817. https://doi.org/10.1093/jxb/erw397

Molina-Montenegro MA, Acuña-Rodríguez IS, Flores TSM, Hereme R, Lafon A, Atala C, Torres-Díaz C (2018) Is the success of plant invasions the result of rapid adaptive evolution in seed traits? Evidence from a latitudinal rainfall gradient. Front Plant Sci 9:208. https://doi.org/10.3389/fpls.2018.00208

Molinari N, D'Antonio C, Thomson G (2007) Carpobrotus as a case study of the complexities of species impacts. In: Cuddington K, Byers JE, Wilson WG, Hastings A (eds) Ecosystem engineers: plants to protists. Elsevier, London, pp 139-162. https://doi.org/ 10.1016/S1875-306X(07)80009-8

Moreira X, Abdala-Roberts L, Linhart YB, Mooney KA (2014) Masting promotes individual- and population-level reproduction by increasing pollination efficiency. Ecology 95:801-807. https://doi. org/10.1890/13-1720.1

Novoa A, González L (2014) Impacts of Carpobrotus edulis (L.) NE $\mathrm{Br}$. on the germination, establishment and survival of native plants: a clue for assessing its competitive strength. PLoS ONE. https://doi.org/10.1371/journal.pone.0107557

Novoa A, González L, Moravcová L, Pyšek P (2013) Constraints to native plant species establishment in coastal dune communities invaded by Carpobrotus edulis: Implications for restoration. Biol Cons 164:1-9. https://doi.org/10.1016/j.biocon.2013.04.008

Oksanen J, Blanchet GF, Friendly M, Kindt R, Legendre P, McGlinn D, Minchin PR, O'Hara RB, Simpson GL, Solymos P, Stevens MHH, Szoecs E, Wagner H (2019) vegan: Community Ecology Package. R package version 2.5-6. https://CRAN.R-project.org/ package $=$ vegan

Poschlod P, Abedi M, Bartelheimer M, Drobnik J, Rosbakh S, Saatkamp A (2013) Seed ecology and assembly rules in plant communities. In: van der Maarel E (ed) Vegetation ecology. WileyBlackwell, Chichester, pp 164-202. https://doi.org/10.1002/97811 18452592.ch6
Preston CD, Sell PD (1988) The Aizoaceae naturalized in the British Isles. Watsonia 17(3):217-245

Pyšek P, Richardson DM (2007) Traits associated with invasiveness in Alien plants: where do we stand? In: Nentwig W (ed) Biological invasions, vol 193. Springer Verlag, Berlin. https://doi.org/10. 1007/978-3-540-36920-2_7

R Core Team (2020) R: a language and environment for statistical computing. R Foundation for Statistical Computing, Vienna. https:// www.R-project.org/

Ranal MA, Santana DG (2006) How and why to measure the germination process? Revista Brasileira De Botanica 29(1):1-11. https:// doi.org/10.1590/S0100-84042006000100002

Roiloa SR, Rodriguez-Echeverría S, López-Otero A, Retuerto R, Freitas H (2014) Adaptive plasticity to heterogeneous environments increases capacity for division of labor in the clonal invader Carpobrotus edulis (Aizoaceae). Am J Bot 101:1301-1308. https:// doi.org/10.3732/ajb.1400173

Roiloa SR, Retuerto R, Campoy JG, Novoa A, Barreiro R (2016) Division of labor brings greater benefits to clones of Carpobrotus edulis in the non-native range: evidence for rapid adaptive evolution. Front Plant Sci 7(394):1-13. https://doi.org/10.3389/fpls. 2016.00349

Ruffino L, Krebs E, Passetti A, Aboucaya A, Affre L, Fourcy D, Lorvelec O, Barcelo A, Berville L, Bigeard N, Brousset L, De Méringo H, Gillet P, Le Quilliec P, Limouzin Y, Médail F, Meunier JY, Pascal M, Ponel P, Rifflet F, Santelli C, Buisson E, Vidal E (2015) Eradications as scientific experiments: progress in simultaneous eradications of two major invasive taxa from a Mediterranean island. Pest Manage Sci 71:189-198. https://doi.org/10. 1002/ps.3786

Saatkamp A, Poschlod P, Venable DL (2014) The functional role of soil seed banks in natural communities. In: Gallagher RS (ed) Seeds - the ecology of regeneration in plant communities. CABI, Wallingford, pp 263-294

Saatkamp A, Cochrane A, Commander L, Guja L, Jimenez-Alfaro B, Larson J, Nicotra A, Poschlod P, Silveira FAO, Cross A, Dalziell EL, Dickie J, Erickson TE, Fidelis A, Fuchs A, Golos PJ, Hope M, Lewandrowski W, Merritt DJ, Miller BP, Miller R, Offord CA, Ooi MKJ, Satyanti A, Sommerville KD, Tangney R, Tomlinson S, Turner S, Walck JL (2019) A research agenda for seed-trait functional ecology. New Phytol 221:1764-1775. https://doi.org/ 10.1111/nph.15502

Sallon S, Solowey E, Cohen Y, Korchinsky R, Egli M, Woodhatch I, Simchoni O, Kislev M (2008) Germination, genetics, and growth of an ancient date seed. Science 320(5882):1464. https://doi.org/ 10.1126/science. 1153600

Santelices Moya R, Espinoza Meza S, Magni Díaz C, Cabrera Ariza A, Donoso Calderón S, Peña Rojas K (2017) Variability in seed germination and seedling growth at the intra- and interprovenance levels of Nothofagus glauca (Lophozonia glauca), an endemic species of Central Chile. NZ J Forest Sci 41:10. https://doi.org/ 10.1186/s40490-017-0091-5

Sattler SE, Gilliland LU, Magallanes-Lundback M, Pollard M, DellaPenna D (2004) Vitamin E is essential for seed longevity and for preventing lipid peroxidation during germination. Plant Cell 16:1419-1432. https://doi.org/10.1105/tpc.021360

Schuetzenmeister A, Dufey F (2020) VCA: Variance Component Analysis. R package version 1.4.3. https://CRAN.R-project.org/ package $=\mathrm{VCA}$

Segura F, Vicente MJ, Franco JA, Martínez-Sánchez JJ (2015) Effects of maternal environmental factors on physical dormancy of Astragalus nitidiflorus seeds (Fabaceae), a critically endangered species of SE Spain. Flora Morphol, Dist, Funct Ecol Plants 216:71-76. https://doi.org/10.1016/j.flora.2015.09.001

Snell RS, Beckman NG, Fricke E, Loiselle BA, Carvalho CS, Jones LR, Lichti NI, Lustenhouwer N, Schreiber S, Strickland C, 
Sullivan LL, Cavazos BR, Giladi I, Hastings A, Holbrook K, Jongejans E, Kogan O, Montaño-Centellas F, Rudolph J, Rogers HS, Zwolak R, Schupp E (2019) Consequences of intraspecifc variation in seed dispersal for plant demography, communities, evolution and global change. AoB PLANTS. https://doi.org/10. 1093/aobpla/plz016

Tielbörger K, Petrů M, Lampei C (2011) Bet-hedging germination in annual plants: a sound empirical test of the theoretical foundations. Oikos 121:1860-1868. https://doi.org/10.1111/j.1600-0706. 2011.20236.x

Tigano A, Friesen VL (2016) Genomics of local adaptation with gene flow. Mol Ecol 25:2144-2164. https://doi.org/10.1111/mec.13606

Traveset A, Moragues E, Valladares F (2008) Spreading of the invasive Carpobrotus spp. in Mediterranean ecosystems: the advantage of performing well in different light environments. Appl Veg Sci 11:45-54. https://doi.org/10.3170/2007-7-18303

Vilà M, Valladares F, Traveset A, Santamaría L, Castro P (eds) (2008) Invasiones biológicas. CSIC, Madrid

Walters C, Wheeler LM, Grotenhuis JM (2005) Longevity of seeds stored in gene bank: species characteristics. Seed Sci Res 15:1-20. https://doi.org/10.1079/SSR2004195
Wen B (2019) Seed germination ecology of Alexandra palm (Archontophoenix alexandrae) and its implication on invasiveness. Sci Rep 9:4057. https://doi.org/10.1038/s41598-019-40733-0

Wickham H (2016) ggplot2: elegant graphics for data analysis. Springer-Verlag, New York. https://doi.org/10.1007/ 978-0-387-98141-3

Willi Y, Van Burskirk J (2019) A practical guide to the study of distribution limits. Am Nat 193(6):773-785. https://doi.org/10.1086/ 703172

Williams NSG, Hahs AK, Vesk PA (2015) Urbanisation, plant traits and the composition of urban floras. Perspect Plant Ecol, Evol Syst 17:78-86. https://doi.org/10.1016/j.ppees.2014.10.002

Wright IJ, Reich PB, Cornelissen JHC, Falster DS, Groom PK, Hikosaka K, Lee W, Lusk CH, Niinemets Ü, Oleksyn J, Osada N, Poorter H, Warton DI, Westoby M (2005) Modulation of leaf economic traits and trait relationships by climate. Glob Ecol Biogeogr 14:411-421. https://doi.org/10.1111/j.1466-822x.2005.001 